ENCYCLOPEDEE Encyclopédie berbère

BERBERE

$11 \mid 1992$

11 | Bracelets - Caprarienses

\title{
Canaries (Îles)
}

E.B. et J. Onrubia-Pintado

\section{(2) OpenEdition}

Journals

Édition électronique

URL : http://journals.openedition.org/encyclopedieberbere/2046

DOI : 10.4000/encyclopedieberbere.2046

ISSN : 2262-7197

\section{Éditeur}

Peeters Publishers

\section{Édition imprimée}

Date de publication : 1 septembre 1992

Pagination : 1731-1755

ISBN : 2-85744-581-4

ISSN : 1015-7344

\section{Référence électronique}

E.B. et J. Onrubia-Pintado, « Canaries (Îles) », Encyclopédie berbère [En ligne], 11 | 1992, document C16, mis en ligne le 01 avril 2013, consulté le 25 septembre 2020. URL : http://journals.openedition.org/ encyclopedieberbere/2046 ; DOI : https://doi.org/10.4000/encyclopedieberbere.2046

Ce document a été généré automatiquement le 25 septembre 2020

(C) Tous droits réservés 


\title{
Canaries (Îles)
}

\author{
E.B. et J. Onrubia-Pintado
}

1 Les Canaries, ancrées au large de la lisière septentrionale du Sahara adantique, peuvent être considérées comme une extension insulaire, marginale et fortement individualisée, $d u$ vaste univers berbère. La présence aux îles de groupes berbérophones du moins dans la phase finale de la préhistoire locale, les temps préhispaniques, semble fermement attestée par plusieurs arguments d'ordre archéologique, ethnohistorique et paléo-linguistique. Mais à l'heure actuelle des difficultés méthodologiques et les interprétations divergentes subsistent en ce qui concerne la détermination de la genèse, la chronologie, la dispersion géographique et l'apparentement linguistique précis de ces populations.

\section{Le milieu physique : écosystèmes actuels et paléo environnements}

2 L'Archipel canarien, composé de sept îles principales et de plusieurs îlots, est situé à une centaine de kilomètres à l'ouest du Cap Juby, entre $27^{\circ} 37^{\prime}$ et $29^{\circ} 24^{\prime}$ de latitude $\mathrm{N}$. Depuis ce promontoire, l'île de Fuerteventura est visible sous des conditions météorologiques exceptionnelles. La structure de la croûte des Canaries, encore imparfaitement connue sur le plan volcanique et pétrographique, suit deux alignements tectoniques principaux qui correspondent à la dorsale atlantique et au massif de l'Atlas. Ces îles illustrent assez bien de nombreux types de morphologies volcaniques, dont les affleurements les plus anciens ne remontent pas au-delà de 40 millions d'années. Les éruptions volcaniques plus récentes sont, elles, d'âge historique voire actuelle. Il est certain que le caractère volcanique de l'archipel conditionne l'entité et la distribution des ressources naturelles telles que l'eau, les sols agricoles ou la pêche.

3 Les Canaries attestent actuellement un caractère écologique hybride, présentant en même temps des traits typiques des régions sahariennes et des propriétés spécifiques des territoires nettement atlantiques. Si du point de vue climatique les îles orientales, Lanzarote et Fuerteventura, peuvent être identifiées à des satellites insulaires du bassin 
de Tarfaya-Laâyoune, désert atténué qui s'étend de l'embouchure de l'oued Drâa à la basse Séguiet el-Hamra, les îles montagneuses du groupe centro-occidental, soumises à l'influence de l'alizé, fournissent, quant à elles, une grande variété de micro-climats. Ceux-ci s'échelonnent en hauteur, en conditionnant la distribution des étages biologiques et des écosystèmes qui leur sont associés. Dans cette moitié occidentale, les côtes et les versants septentrionaux, zones de condensation de l'alizé, offrent des territoires humides favorables aux installations humaines. A présent, les conditions physiques de navigation dans les eaux canario-sahariennes sont commandées, dans une large mesure, par les caractéristiques atmosphériques et océanographiques de cette région: l'alizé, la houle du large et le courant des Canaries. D'autre part, les changements soudains de la direction des vents dominants et le caractère abrupt des côtes, certainement lié à l'existence de calmes persistants dans les canaux interinsulaires et à la rareté d'abris et de mouillages adéquats, rendent difficiles les parcours maritimes dans la Mer des Canaries.

4 Les premières sources écrites, faisant incontestablement référence à l'Archipel canarien, semblent décrire, au xve siècle, un «paysage naturel » différent de l'actuel. Mais la définition de l'ancien cadre écologique de cette aire géographique est encore largement tributaire d'extrapolations souvent risquées. Si les observations et leur contexte empirique paraissent unanimement acceptés, il n'en est pas de même, en revanche, quant à l'interprétation de leurs interactions causales et à la détermination de leurs implications. Les lacunes restent nombreuses, la pertinence du morcellement de l'écosystème est fort souvent omise, la chronologie des événements climatiques demeure hétérogène et contestée et les hypothèses explicatives sont divergentes voire contradictoires. Cependant, nul ne doute que l'intérêt des recherches écologiques et biogéographiques portant sur des aires insulaires telles que l'Archipel canarien n'est pas négligeable pour la compréhension de l'évolution du peuplement humain de ces territoires. L'espace géographique fermé, l'isolement relatif, la limitation des ressources, la réduction significative de la concurrence extérieure et, par conséquent, la préservation de certains traits archaïques, toujours en compétition avec une vulnérabilité et instabilité extrêmes, sont autant de propriétés spécifiques aux écosystèmes des îles et à la personnalité des cultures insulaires. Ces caractéristiques environnementales esquissent un tableau de corrélations où s'insèrent, par le biais des particularités adaptatives propres aux groupes humains, les stratégies de subsistance, les formules d'acquisition économique, les modes d'occupation du territoire et, en somme, la dynamique du changement culturel.

\section{La préhistoire canarienne : mise en place des berbères insulaires}

Les Iles Canaries, restées à l'écart des courants civilisateurs et commerciaux jusqu'au XIV ${ }^{e}$ siècle, malgré les incursions probables des navigateurs de l'Antiquité et du Haut Moyen Age, constituent une sorte d'isolat culturel où chaque unité insulaire semble se comporter comme un authentique micro-continent. L'insularité joue ainsi dans ce territoire le rôle de facteur retardataire et explique de manière satisfaisante l'archaïsme déconcertant qui frappa les premiers navigateurs européens arrivés dans l'archipel. Les expéditions européennes les plus précoces et la conquête définitive des Canaries, qui se traduit par une forte acculturation atteignant parfois les limites du 
véritable ethnocide, marquent la fin des temps préhispaniques insulaires et l'incorporation de ces îles aux systèmes socio-économiques du monde occidental à la fin du Moyen Age. Le processus d'affrontement traumatique, dissolution et assimilation des sociétés indigènes canariennes, constitue de telle sorte un point de repère fondamental pour l'étude des motivations économiques et idéologiques de la colonisation européenne de l'Afrique atlantique, ainsi que pour la détermination du sens de l'évolution et des changements structuraux des sociétés dites "primitives » au contact du monde "civilisé ». La préhistoire de l'Archipel canarien, au sens large du terme, s'étale donc depuis le moment encore fort imprécis où s'est produit le peuplement initial des îles jusqu'au $\mathrm{Xv}^{\mathrm{e}}$ siècle.

Bâtons et armes en bois des Guanches de Ténérife

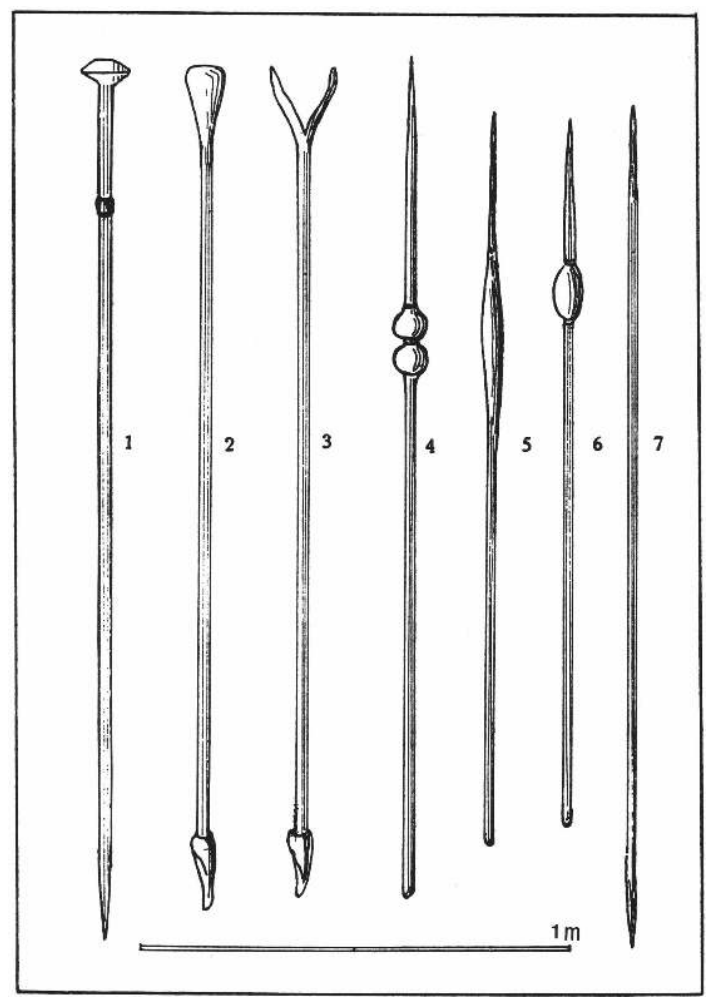

1 : canne de commandement (anpa) ; 2 et 3 : houlettes avec extrémité en corne ; 4 à 6 : javelots (banotes) ; 7 : lance, d'après D. Cuzcoy.

6 Les anciens habitants des Iles Canaries, dénommés à tort guanches car ce qualificatif convient sensu stricto aux indigènes préhispaniques de Ténérife, se comportent dans leur ensemble comme des groupes fortement marginaux où les survivances culturelles sont de toute évidence le trait le plus pertinent. Cette caractéristique accorde des connotations spécifiques à cet univers insulaire tout en demandant une approche méthodologique particulière. Mis à part quelques précieux renseignements d'ordre ethnohistorique, socio-économique et culturel fournis par les sources européennes, qui feront l'objet d'un commentaire détaillé plus loin, l'essentiel des données nécessaires pour esquisser un tableau approximatif des Canaries préhispaniques est emprunté à deux disciplines : l'anthropobiologie et l'archéologie. 


\section{L'anthropobiologie}

7 Les premières études anthropologiques, menées notamment par R. Verneau à partir de la fin du siècle dernier, contribuèrent à éveiller un intérêt précoce pour les populations canariennes anciennes. Dans ses travaux, le Dr Verneau reconnaissait plusieurs types humains, en dehors du «type guanche » qui présentait des caractères cromagnoïdes nets. Il est donc apparu très tôt que le peuplement préhistorique des Iles Canaries n'était pas uniforme. Paradoxalement la généralisation progressive de l'emploi du terme guanche à l'ensemble de tous les habitants préhispaniques de l'Archipel canarien, par delà toute considération physique, allait donner une impression contraire.

8 A la suite de Verneau, divers auteurs ont insisté sur cette hétérogénéité physique en multipliant de façon excessive les anciens «types " anthropologiques canariens. Après avoir ainsi longtemps méconnu en paléoanthropologie une donnée élémentaire, celle de la variabilité individuelle au sein d'une population, pour privilégier une conception essentiellement construite sur un système typologique multipliant les « holotypes », les paléoanthropologues sont maintenant revenus à des notions plus simples sur la nature physique de ce peuplement préhistorique canarien, en reconnaissant, notamment à la suite des travaux de I. Schwidetzky et M. Fusté, deux composantes essentielles: cromagnoïde et protoméditerranéenne.

9 Il faut remarquer d'emblée que cette dualité physique est justement celle observée dans le peuplement préhistorique du Maghreb et qu'on la trouve, au fur et à mesure des nouvelles découvertes, dans de nombreux autres sites préhistoriques d'Afrique septentrionale, saharienne et nord-sahélienne, sous la forme d'une dichotomie physique entre cromagnoïdes et non-cromagnoïdes. L'origine de la composante cromagnoïde, la première identifiée aux Canaries, suscite encore de nombreuses interrogations, certains auteurs ayant même émis l'hypothèse d'un phénomène de convergence évolutive, les traits cromagnoïdes des anciens canariens ayant été acquis par des processus d'évolution sur place, particuliers à ce milieu clos. Cependant cette théorie n'est pas acceptée et, pour la majorité des spécialistes, l'authenticité des cromagnoïdes canariens ne fait aucun doute et l'origine de leur morphologie est extérieure à l'archipel. Le «type guanche» de Verneau a donc été rapproché des individus mechtoïdes qui constituent le support humain exclusif des industries ibéromaurusiennes du Maghreb. Des découvertes récentes ont par ailleurs montré la présence, à l'Holocène moyen, de représentants de ce groupe de Mechta-Afalou sur le littoral du bassin de Tarfaya, l'hinterland continental des Canaries. Quant au type protoméditerranéen, le type II de Verneau, il est, lui, très largement représenté en Afrique du Nord, mais aussi dans tout le bassin méditerranéen. Les individus protoméditerranéens semblent intimement liés à l'apparition des industries capsiennes au Maghreb, où ils sont attestés pendant toute la durée des temps préhistoriques et historiques à partir de cette civilisation épipaléolithique. Il n'est peut-être pas sans intérêt de rappeler ici que certains auteurs font coïncider, de façon intuitive certes, l'irruption de cette culture en Afrique du Nord avec la mise en place des premières populations berbérophones. Néanmoins on connaît encore mal la composition exacte de ce peuplement canarien non-cromagnoïde qui n'est probablement pas homogène, mais qui s'individualise dans son ensemble en s'opposant aux caractères physiques très particuliers des cromagnoïdes. 
10 Bien que les données anthropologiques attribuent à chacun des complexes ethniques insulaires une personnalité accentuée, il paraît possible d'établir toutefois certaines relations typologiques entre les populations préhistoriques des différentes îles. D'une part, les études des années cinquante et soixante, aujourd'hui devenues classiques, avaient déjà distingué un groupe insulaire central plutôt cromagnoïde et un ensemble périphérique, composé des populations de la Grande Canarie, Fuerteventura, La Palma et $\mathrm{El} \mathrm{Hierro,} \mathrm{en} \mathrm{grande} \mathrm{partie} \mathrm{méditerranéen.} \mathrm{D'autre} \mathrm{part,} \mathrm{des} \mathrm{dispersions} \mathrm{régionales}$ inverses avaient été identifiées, au même moment, entre les effectifs cromagnoïdes et protoméditerranéens de Ténérife et de la Grande Canarie, le type protoméditerranéen étant majoritaire dans la région septentrionale en Grande Canarie et dans la zone méridionale à Ténérife. Des travaux plus récents, portant en particulier sur la dentition des populations fossiles canariennes, sont venus étayer les équivalences et les dissymétries anthropo-géographiques proposées. Si l'analogie des individus de Ténérife et La Gomera semble corroborée, il n'en est pas de même quant au contraste entre les groupes septentrionaux et méridionaux de Ténérife, la denture de l'ensemble des populations préhistoriques de cette île étant tout à fait homogène. Par ailleurs, la dentition des individus de l'intérieur de la Grande Canarie, souvent assimilés aux cromagnoïdes de Ténérife et La Gomera, est nettement distincte des séries dentaires des populations préhispaniques de ces îles.

11 En plus des déterminations typologiques, les études anthropologiques ont mis en évidence plusieurs données que l'on pourrait qualifier de socio-économiques: variabilité individuelle et implications sociales des différents rites funéraires (inhumations, momifications), identification de certains noyaux de populations endogames, établissement de contrastes des régimes alimentaires préhispaniques.

Il faut, en conclusion, rejeter l'idée que le peuplement préhistorique canarien se contente simplement de reproduire le modèle continental. Le milieu insulaire offre en effet pour l'évolution des espèces de nombreuses particularités auxquelles l'homme ne saurait échapper, parmi lesquelles divers phénomènes dépendant de l'isolement géographique (dérive génétique, effectifs réduits, consanguinité élevée, effet de fondateurs). Il est évident que de nombreuses imprécisions persistent sur le plan paléoanthropologique, notamment: relations morphologiques exactes entre les cromagnoïdes canariens et continentaux, importance de la variabilité morphologique de l'élément cromagnoïde, pertinence des phénomènes continentaux ou insulaires de métissage entre populations cromagnoïdes et groupes protoméditerranéennes et, enfin, répartition géographique précise de deux éléments anthropologiques dans l'archipel. Malheureusement les limitations méthodologiques des collections ostéologiques disponibles, liées pour l'essentiel à l'ancienneté des ramassages, ne permettent pas de fournir des réponses, aussi approximatives soient-elles, à ces questions. Les séries anthropologiques canariennes, quantitativement très importantes, ne sont pas significatives du point de vue statistique (restes d'enfants peu abondants, représentation géographique différente), leur provenance, leur contexte archéologique et leur attribution chronologique faisant très souvent défaut. 
Céramique guanche de Ténérife : vases à décor incisé et poinçonné et vases à anses-verseuses, d'après D. Cuzcoy.
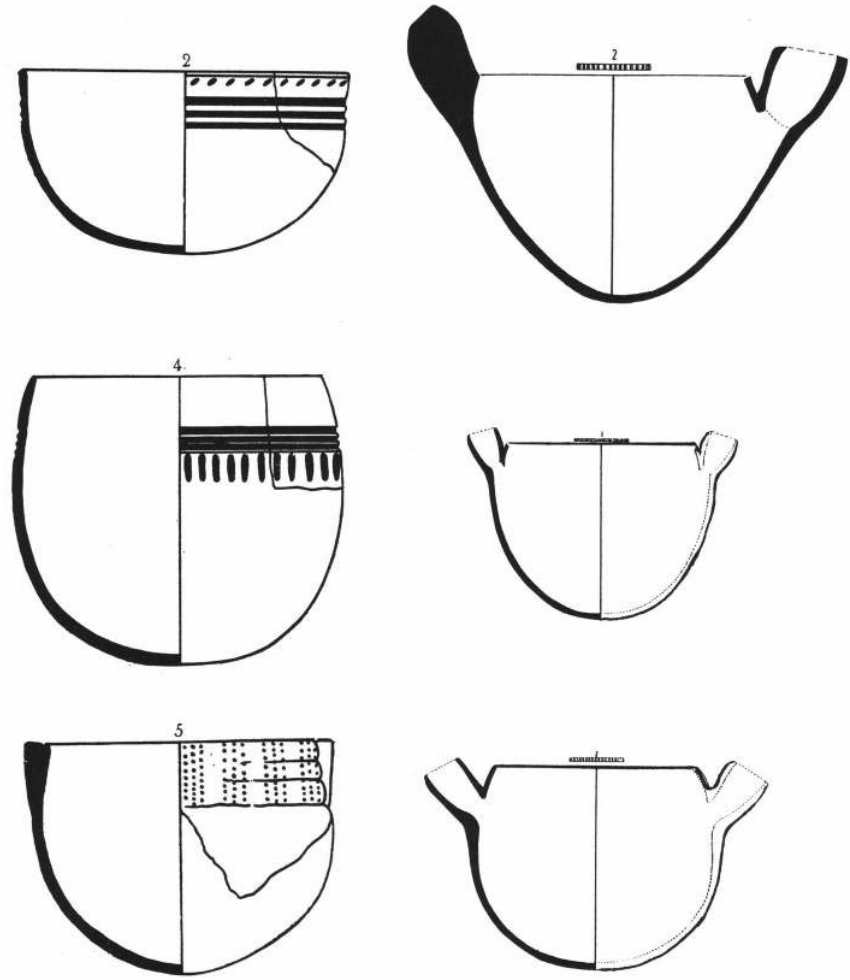

\section{L'archéologie}

13 A l'heure actuelle, la plupart des spécialistes ayant abordé le problème de l'origine du peuplement préhistorique canarien sont apparemment d'accord sur un double axiome : son caractère polygénétique africain sensu lato et sa chronologie tardive, ingénument qualifiée de post-néolithique. Les arguments plutôt intuitifs qui soutiennent ces énoncés sont tirés de la diversité interinsulaire et des séries des datations absolues disponibles. Mais il est paradoxal de constater la façon dont l'hyper-diffusionnisme des modèles "invasionnistes " frôle la lecture évolutionniste et souvent univoque des séquences culturelles insulaires. Le peuplement de chacune des îles et les changements culturels majeurs sont volontiers mis en rapport étroit avec des «stimulus » divers dont le point de départ présumé est parfois éloigné de plusieurs milliers de kilomètres, les jalons intermédiaires éventuels faisant défaut. En revanche, l'existence de rapports préhistoriques inter-insulaires et, par conséquent, d'une homogénéité culturelle partielle est difficilement acceptée même pour les îles voisines. Quant aux attributions chronologiques, fréquemment les datations par recoupements sont utilisées, par le biais de l'archéologie comparée, au même titre que les dates absolues.

La remarquable différence inter-insulaire sur le plan archéologique fut mise en évidence déjà au siècle dernier. Les études plus récentes n'ont fait que confirmer, à partir d'une approche archéographique dépourvue de profondeur chronologique, le caractère hétérogène des cultures préhistoriques canariennes. Même la "culture de substrat ", ce fond pancanarien que l'on avait cru saisir à l'origine de la présence humaine dans l'archipel, et dont l'existence paraissait indispensable pour émettre une hypothèse raisonnable quant au processus de fréquentation et colonisation des îles, 
semble devenir flou au fur et à mesure que les recherches se multiplient. Cette pluralité, caractéristique des milieux insulaires complexes, doit être mise en parallèle avec des environnements divers et des particularités adaptatives. Mais il est indéniable qu'à l'aube des temps préhistoriques canariens ni l'impact peut-être sélectif d'éléments allochtones ni les relations inter-insulaires ne peuvent être exclus des composantes formatives des différents faciès culturels canariens. De ce point de vue, l'argument de la contiguïté, du " voisin le plus proche ", doit être retenu en ce qui concerne la direction et le module des vecteurs hypothétiques du peuplement humain de l'Archipel canarien.

Tumulus de la Guancha, monument funéraire collectif, à Gaïdar (Grande Canarie).

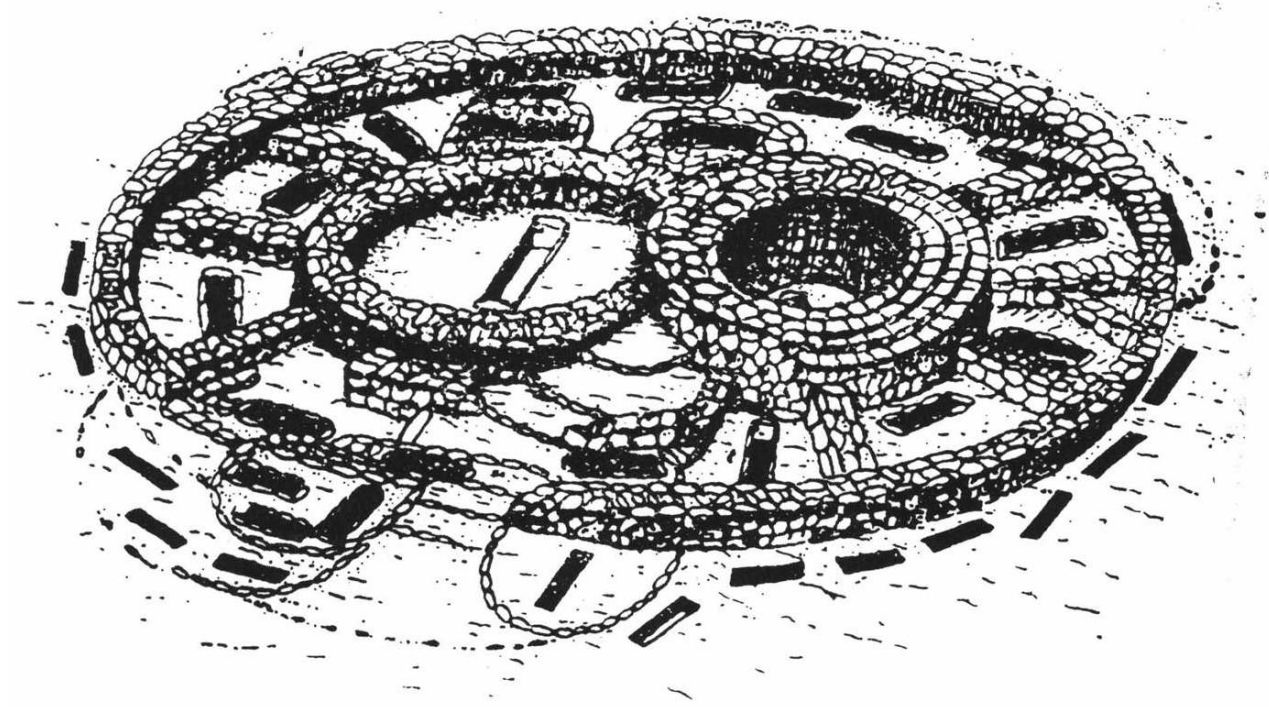

15 Les problèmes posés par la chronologie des cultures préhistoriques canariennes sont certes notables. Les séries de dates radiométriques, discrètement fiables dans de nombreux cas, font remonter le témoignage le plus ancien d'une présence humaine dans l'archipel à $2490 \pm 60 \mathrm{BP}$ (environ $\mathrm{vI}^{\mathrm{e}}$ siècle av. J.-C). Il s'agit du niveau inférieur de la grotte de La Arena, à Barranco Hondo dans l'île de Ténérife, où des aires de combustion renfermant des os de lacertidés brûlés ont été interprétés comme les traces d'une activité anthropique souvent contestée. Le caractère relativement récent de cette datation semble corroboré par l'utilisation abusive de l'archéologie comparée. Mais les parallèles traditionnellement choisis sont aussi flous qu'insoutenables sur le plan épistémologique et méthodologique. 
Figurines anthropomorphes en terre cuite de la Cueva Pintada à Gaïdar (Grande Canarie).
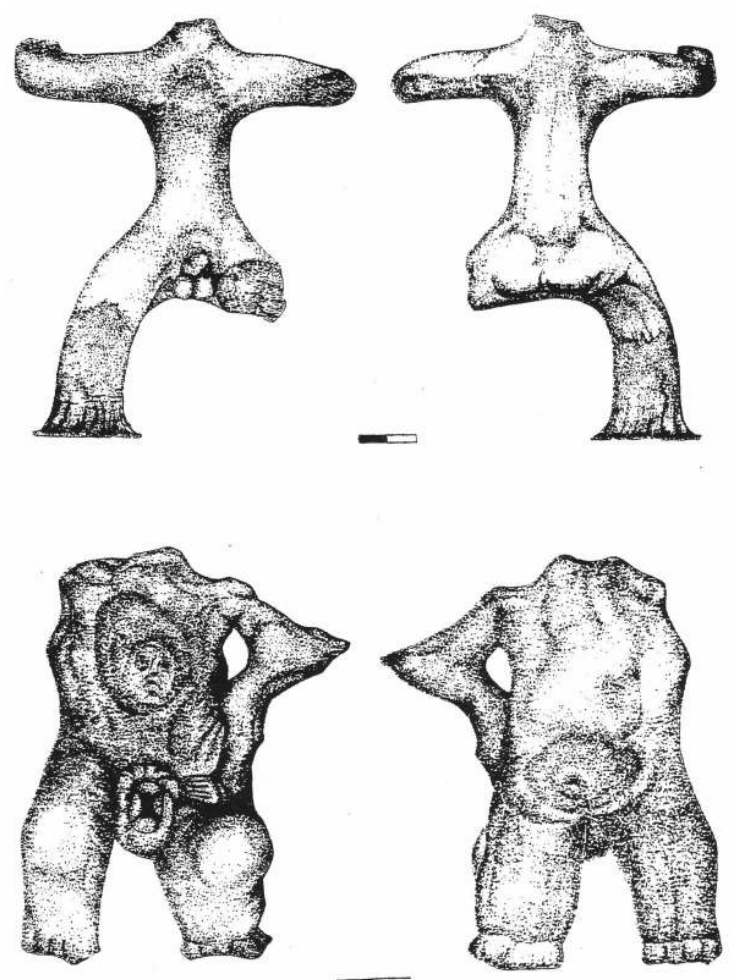

Figurines en terre cuite. En haut et en bas de la Cueva Pintada, au centre de provenance inconnue.
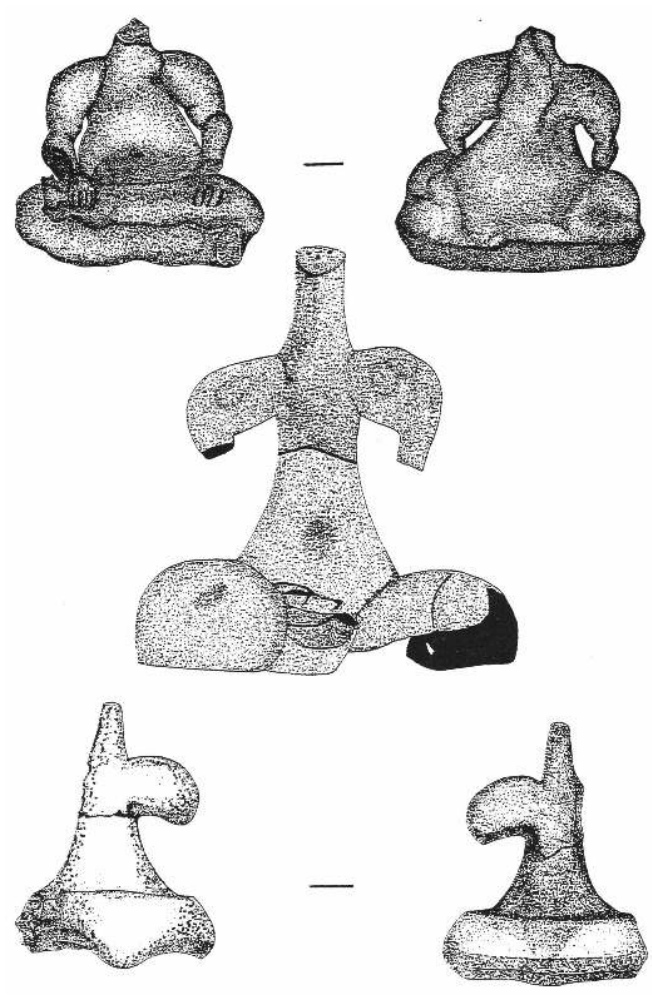

En outre, il va sans dire que dans des milieux fortement marginaux que sont les îles, les phénomènes de convergence, les survivances culturelles et les anachronismes extrêmes 
ont un poids considérable. Si la chronologie absolue atteste une occupation effective de certaines îles, telles que Lanzarote, la Grande Canarie, Ténérife et La Palma, dans la deuxième moitié $\mathrm{du} \mathrm{I}^{\mathrm{er}}$ millénaire av. J.-C, elle ne s'oppose pas à un peuplement plus ancien. Les datations au $14 \mathrm{C}$ plus élevées concernent, sauf dans le cas de la grotte de $\mathrm{La}$ Arena, des horizons culturels où les systèmes économiques de production sont bien affirmés et le succès des stratégies d'adaptation semble garanti depuis un laps de temps impossible à évaluer. Dans le cadre d'un modèle archéologique insulaire, il est difficilement admissible que cette absence de tâtonnements adaptatifs puisse correspondre à une phase initiale de la colonisation de l'archipel. Cette conviction est d'autant plus pertinente que les îles orientales ont livré des dates globalement plus récentes que celles de l'archipel occidental, contrairement à ce que l'on aurait pu attendre si l'on postule, comme il paraît raisonnable, un peuplement originel progressant d'est en ouest à partir de l'hinterland continental.

Poteries peintes et pintaderas de la Cueva Pintada à Gaïdar (Grande Canarie).

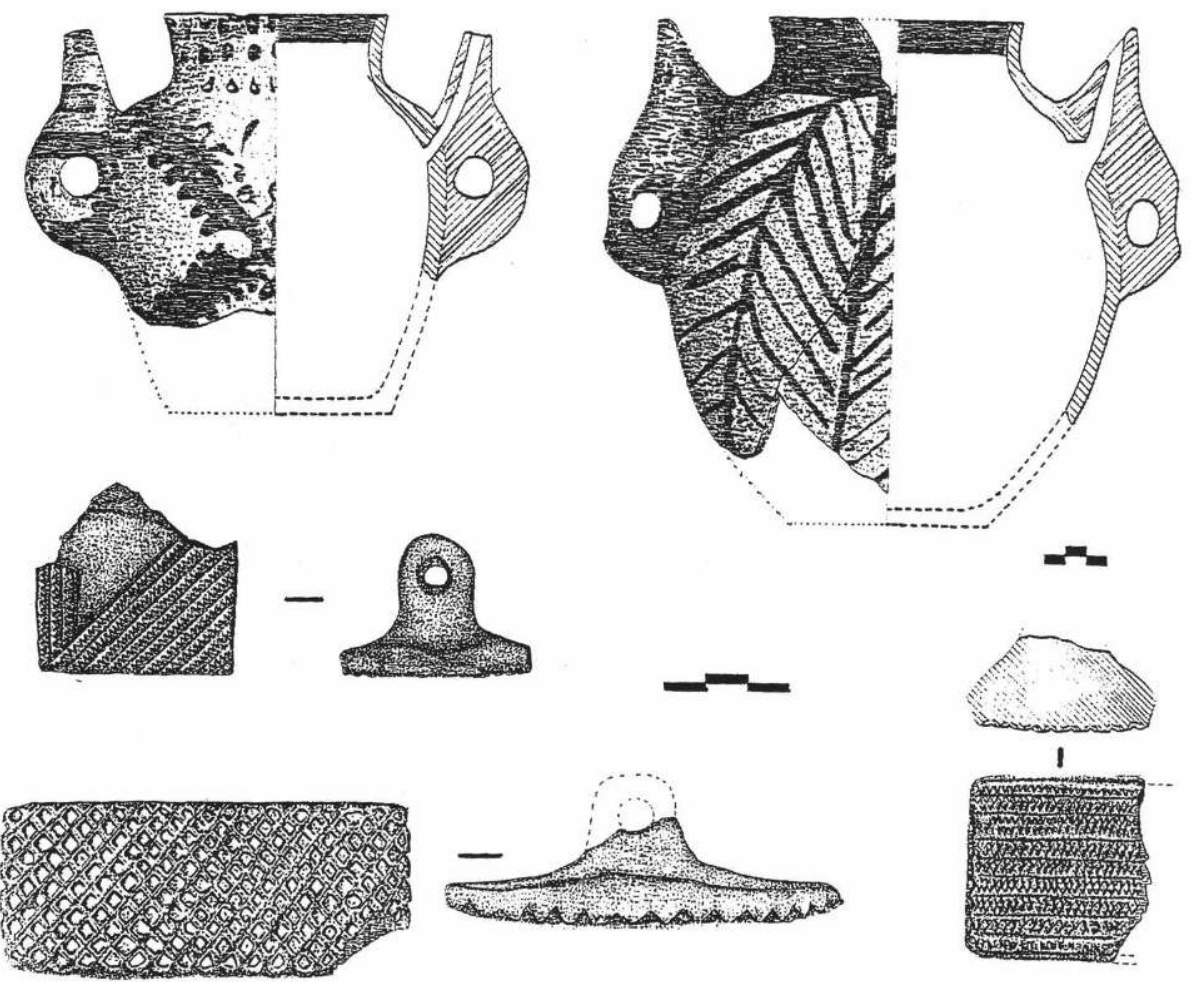

17 Tous les éléments chronologiques indirects aujourd'hui disponibles portent à penser que le peuplement de l'archipel oriental par le "groupe canarien ancestral», dont la volonté colonisatrice ne fait guère de doute, a pu avoir lieu vers la fin du II $^{\mathrm{e}}$ millénaire avant notre ère. Ce groupe canarien ancestral doit être plutôt interprété en termes de génétique des populations qu'identifié à des répertoires archéologiques qui constitueraient une éventuelle "culture de substrat» ou "fond pancanarien». La compréhension des mécanismes de colonisation des Iles Canaries paraît donc largement tributaire de la détermination de la portée réelle et des implications spatiales des phénomènes tels que les effets de fondateurs, la dérive génétique, l'évolution des effectifs ou le stress démographique. Mais ces facteurs ne sont pas strictement naturels. Bien au contraire, ils sont la résultante de l'interaction d'un ensemble de variables physico-biologiques et culturelles : environnement écologique, 
patrimoine génétique, adaptation culturelle et sociale, comportement, perception du territoire...

Poteries incisées de l'lle de La Palma.
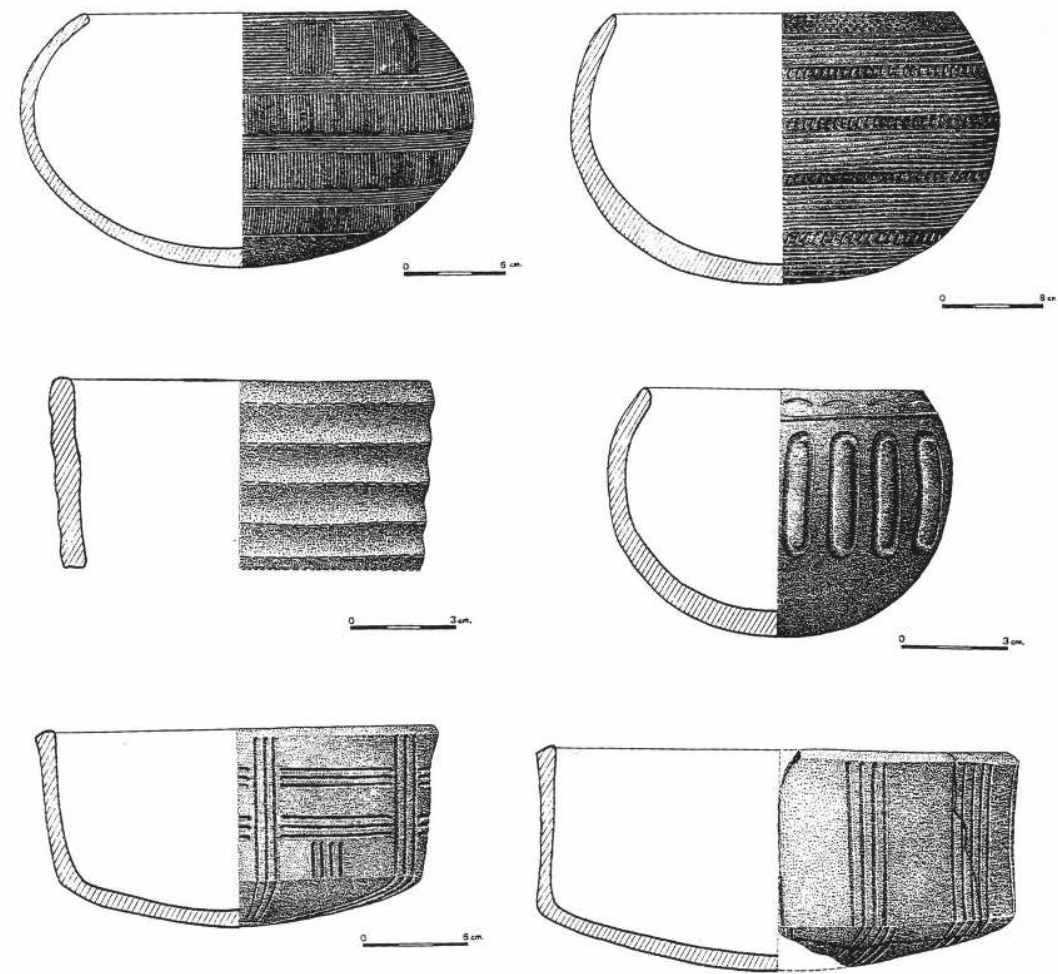

Poteries incisées de Fuerteventura.

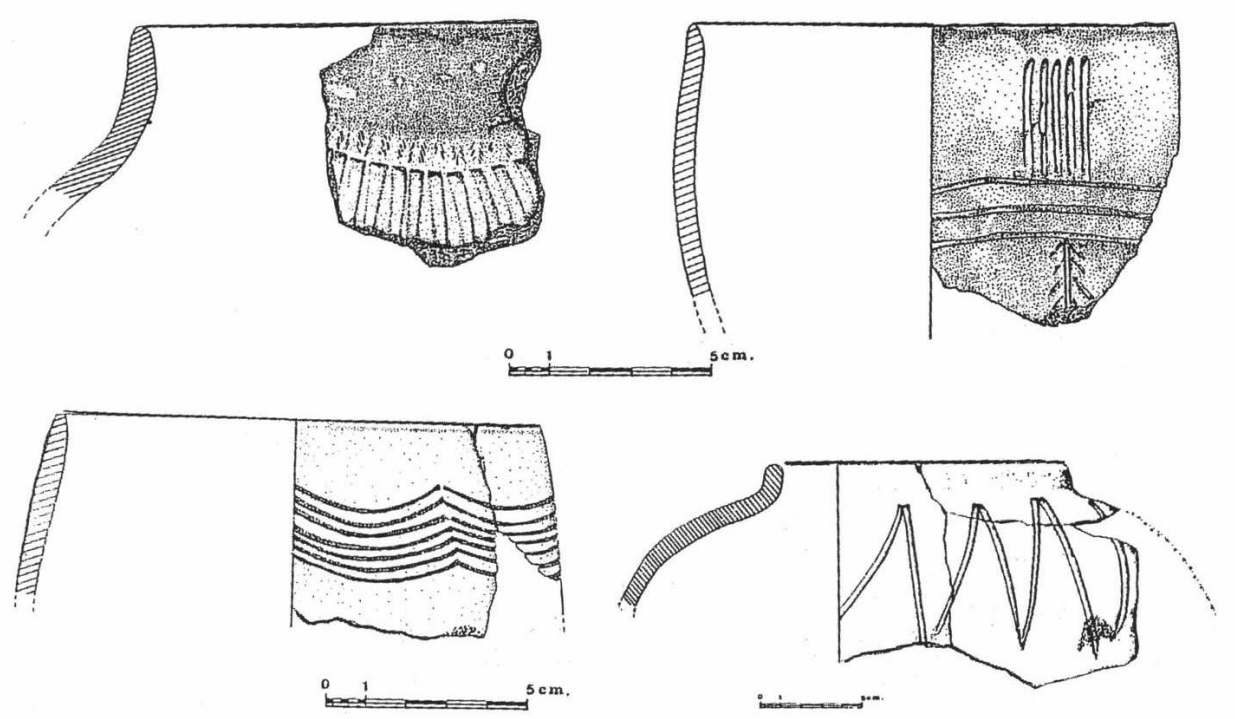

Les éléments archéologiques susceptibles d'être associés à cet horizon archaïque de peuplement ne sont pas facilement saisissables, de même que son support anthropologique virtuel. Premièrement, il semble bien que les occupations humaines les plus précoces demeurent inconnues. D'autre part, l'établissement de filiations culturelles directes n'est tolérable que pour des territoires adjacents dans des 
conditions d'observation extrêmement favorables. Dans les régions insulaires, les limitations environnantes et la restriction des réponses adaptatives peuvent souvent provoquer des phénomènes de convergence sur le plan technologique et socioéconomique difficiles à dépister. L'interprétation des équivalences culturelles simples et isolées doit être donc abordée avec une grande prudence. Finalement, la pertinence de l'aboutissement des modèles dynamiques d'évolution en milieu clos, typiques des écosystèmes des îles, et leur corollaire dans le domaine de l'individualisation culturelle et de la diversité insulaire masquent fréquemment le problème des origines des variables formatives des civilisations préhistoriques canariennes. En Grande Canarie, par exemple, le développement de ces spécificités va de pair avec une complexité socioéconomique croissante qui agit de façon diffuse sur un substrat archaïque préexistant. Ce processus atteint son point culminant, dans la phase finale de la préhistoire locale, dans l'essor d'un authentique paradigme de culture insulaire caractérisé par sa vitalité et sa vocation syncrétique, l'horizon de la Cueva Pintada.

Si l'interprétation du processus de fréquentation et colonisation des différentes îles demeure controversée, le rôle prépondérant, plus ou moins immédiat, des territoires maghrébins et sahariens dans la préhistoire de l'ensemble de l'archipel fait en revanche l'unanimité des spécialistes. Les travaux et monographies publiés depuis les premières synthèses insistent sur cet argument, tout en spécifiant la filiation culturelle éventuelle de chacun des éléments, le nombre de vagues de peuplement, leur intensité par rapport à chaque île, la nature de leur impact et leur chronologie. Tour à tour, les structures d'habitat, les rites et monuments funéraires, le mobilier archéologique et les manifestations d'art rupestre ont été mis en rapport avec des éléments hypothétiquement analogues du Maghreb et du Sahara. Dans ce domaine du comparatisme culturel linéaire, les équivalences avec des modèles communément qualifiés de berbères sont souvent évoquées.

\section{Les inscriptions en caractères libyco-berbères}

20 La seule preuve archéologique de la présence aux Canaries de groupes berbérophones au cours des temps préhispaniques est fournie par les inscriptions alphabétiques en caractères libyques, l'existence de liens directs entre ces écritures et la langue berbère étant presque unanimement acceptée. A présent ces documents épigraphiques, pour l'essentiel de nature rupestre, ont été attestés dans toutes les îles à l'exception de celle de La Gomera. Quoique l'on ne dispose pas actuellement d'un corpus exhaustif des inscriptions canariennes, celles-ci ont été rattachées sans hésitation aux écritures libyco-berbères sahariennes. Certains caractères rapprochent les inscriptions insulaires de l'écriture touarègue actuelle, tandis que d'autres les apparentent à l'alphabet touareg « ancien » plutôt qu'à celui du Sahara occidental.

21 En dépit des complexes problèmes chronologiques liés aux alphabets sahariens, il est certain que les tifinagh actuels sont connus des habitants de l'Ahaggar avant le $v^{e}$ siècle de notre ère, mais il semble que l'introduction de cet alphabet au Sahara remonte au moins au $\mathrm{I}^{\text {er }}$ siècle av. J.-C. Quant à la chronologie des inscriptions libyco-berbères canariennes, leur caractère apparemment tardif du point de vue typologique paraît certifié par deux dates au $14 \mathrm{C}$ situées entre le VIII et le $\mathrm{x}^{\mathrm{e}}$ siècles de notre ère. Ces datations proviennent d'un site funéraire de l'île d'El Hierro, la grotte de Hoyo de los Muertos, dont les cadavres gisaient allongés sur des brancards constitués d'un 
assemblage de planches en bois. L'une de ces planches portait plusieurs signes libycoberbères gravés. La datation absolue de cette inscription convient à la chronologie récente proposée par certains auteurs, à titre d'hypothèse, pour l'irruption des premiers groupes berbérophones en Grande Canarie et à La Palma. Elle conforte aussi la reconstitution glosso-chronologique, intéressante et hasardeuse à plus d'un titre, récemment mise en place par A. Militariev dans le cadre des rapports éventuels des parlers touaregs avec la "langue " préhispanique canarienne, improprement appelée guanche. Toutefois il reste à préciser, dans le domaine des témoignages épigraphiques en caractères libyco-berbères, le rôle des populations maures arrivées à l'Archipel canarien à partir du $\mathrm{xv}^{\mathrm{e}}$ siècle, surtout en ce qui concerne les îles orientales où leur concentration fut extrêmement importante.

Mais si des "graveurs » d'inscriptions libyques sont déjà aux Canaries autour de la fin $\mathrm{du} \mathrm{I}^{\mathrm{er}}$ millénaire ap. J.-C, seules la prudence méthodologique et l'absence de traces matérielles et linguistiques indiscutables empêchent de considérer comme berbérophones les groupes humains qui peuplaient l'archipel avant ce seuil chronologique. La mise en place des paléoberbères dans l'ensemble de l'Afrique du Nord, et notamment dans l'hinterland continental canarien où les vestiges de leur présence sont relativement abondants, semble remonter au-delà de la date plus ancienne actuellement attribuée à la première vague colonisatrice abordant aux îles. De là à envisager un peuplement canarien ancien originairement berbère, il n'y qu'un pas que les données archéologiques disponibles interdisent néanmoins de franchir.

\section{Les sources antiques et arabes : mythes et réalités}

Les Iles des Bienheureux, les Makárôn Nesôi et les Fortunatae Insulae des auteurs grecs et latins, occupent une place privilégiée dans les textes classiques depuis Homère. Mais l'identification de cette contrée fabuleuse située au-delà des Colonnes d'Hercule, et caractérisée par les nourritures aisées, les animaux paisibles et un climat particulièrement doux, avec l'Archipel canarien date assurément des temps hellénistiques.

Parmi les textes antiques relatifs aux Canaries, les références aux relations génétiques, plus ou moins hypothétiques, entre les îles et le continent africain sont tout à fait exceptionnelles. L'important ensemble des périples de l'Antiquité, étudié de façon magistrale par J. Desanges, n'offre aucune précision incontestable sur ce genre de rapports, tout en rendant la reconnaissance de l'archipel extrêmement hasardeuse. Quoique de nombreux auteurs aient cru pouvoir assimiler certaines des îles aux descriptions géographiques du récit d'Hannon, poussés surtout par le besoin technique des bateaux carthaginois de s'éloigner de la côte pour entreprendre le voyage du retour, le caractère problématique de ce texte ne permet pas d'en tirer des conclusions définitives. En tout état de cause, le périple de Polybe (146 av. J.-C), sans aucun doute le document maritime le plus précis que l'on possède sur le littoral atlantique du Maroc actuel, ne parle absolument pas des Canaries. La navigation de Polybe, dont le récit fut transmis par Pline l'Ancien, semble s'arrêter au Cap Juby sans atteindre l'archipel. 


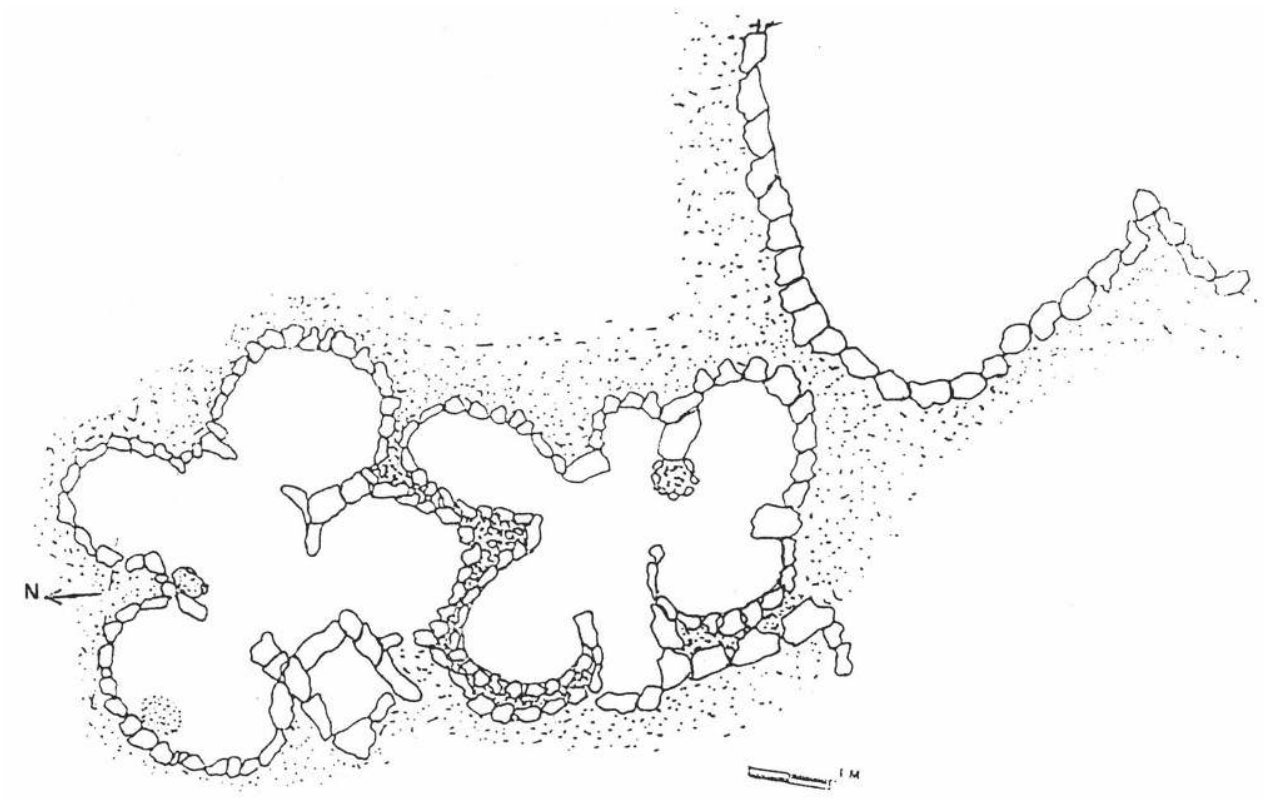

Il paraît probable qu'au cours du $\mathrm{I}^{\mathrm{er}}$ siècle av. J.-C, les marins gaditans, qui fréquentaient depuis longtemps la côte atlantique de la Maurétanie, avaient déjà pris connaissance des Canaries. La biographie de Sertorius, compilée par Plutarque, et des textes de Salluste et Strabon confirment la plausibilité de ce fait. R. Rebuffat établit, tout en évitant soigneusement une quelconque identification géographique, une certaine continuité des parcours des marins de Gades dans les eaux des îles océaniques à partir $d u v^{e}$ siècle avant notre ère. Ces expéditions maritimes des gaditans qui sont peut-être à l'origine de la rivalité étrusco-carthaginoise dans l'Atlantique, à laquelle Diodore fait allusion, se prolongent jusqu'au $\mathrm{I}^{\mathrm{er}}$ siècle av. J.-C. C'est précisément cette marine gaditane qui fait part à Sertorius, pendant son séjour dans le sud de la Péninsule Ibérique, de ses connaissances nautiques qui nous sont parvenues grâce à Salluste et à Plutarque. Le texte de la $\mathrm{XvI}^{\mathrm{e}}$ Epode d'Horace est directement tributaire de cette tradition.

Mais ce n'est que dans l'Historia Naturalis de Pline l'Ancien que l'on trouve la première référence indubitable à un voyage à destination des Canaries. Dans le livre VI $(36,37) \mathrm{de}$ cet ouvrage, le texte antique qui comporte la description la plus précise des îles, on note, à côte de quelques renseignements dûs à Statius Sebosus, le rapport de l'expédition navale envoyée dans l'archipel par le roi Juba II. Ce personnage profondément hellénisé, roi de Maurétanie entre les années 25 av. et 23 ap. J.-C, décide de dépêcher plusieurs embarcations aux Canaries, il y était poussé certainement par des données carthaginoises auxquelles il avait eu accès. Le principal résultat de cette mission de reconnaissance fut celui de la fixation du nombre d'îles qui composaient l'archipel, ainsi que la détermination de la présence de "vestiges constructions » dans plusieurs d'entre elles. C'est à partir de ce récit que Ptolémée va consacrer l'existence d'un ensemble de six îles au large du Sahara dont le souvenir fictif se maintiendra jusqu'à la « redécouverte » européenne de l'archipel. A la fin du Bas Empire, les voyages entre les rivages du détroit de Gibraltar et les Iles Canaries s'interrompent en raison de la grave crise économique et institutionnelle qui s'empare de l'Hispanie et de la 
Maurétanie Tingitane romaines. L'Archipel canarien ne sera donc connu au Haut Moyen Age que par les lecteurs éventuels des auteurs classiques.

Si la connaissance des Canaries chez les historiens et géographes arabes est largement tributaire des données géographiques de Ptolémée et des légendes chrétiennes empruntées aux auteurs hispaniques, il n'est pas impossible d'admettre, certes avec beaucoup de prudence, l'existence de voyages aux îles de la part des marines musulmanes, maghrébines ou andalouses.

Poteries incisées et figurines en terre cuite de Zonzomas (Lanzarote).

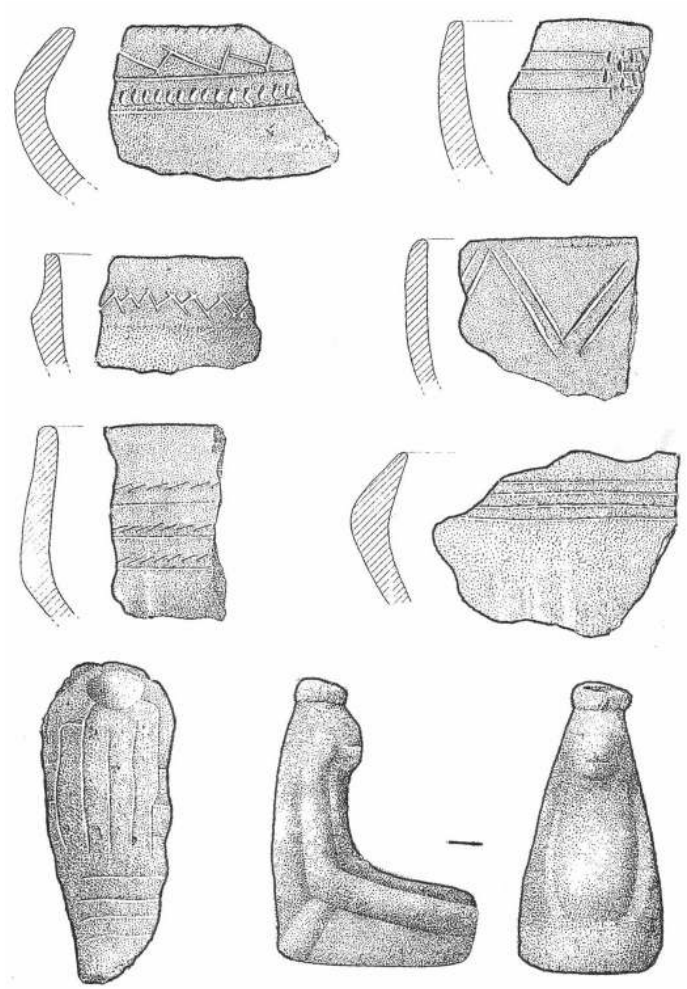

Les Iles Canaries, les Djazā'ir al-Khālidāt des auteurs arabes, sont citées assez souvent par les géographes musulmans à partir du milieu du $\mathrm{IX}^{\mathrm{e}}$ siècle, mais très peu de textes permettent de parler d'une connaissance directe de l'archipel. Au XII siècle, le Kitāb Nuzhat al-muštāa q fi'khtirāq al-āfāq d'El Idrisi et le Kitāb al-istibsāt, largement inspiré d'El Bekri, laissent supposer la réalisation de certaines expéditions aux Canaries menées par des navigateurs almoravides. Le traité de géographie d'El Idrisi rapporte, mises à part d'autres références plus banales, un très curieux voyage: le périple canarien des "aventuriers" de Lisbonne. Le récit de cette navigation des mugarrirum, vraisemblablement authentique quoiqu'enrichie de traditions fabuleuses d'après $T$. Lewicki, semble dépendre d'un texte antérieur. La chronologie approximative de sa réalisation doit être située autour du $x^{e}$ siècle. De la fin du XII ${ }^{e}$ ou des débuts du XII siècles date un texte relatant une hypothétique prédication musulmane aux Canaries. Il s'agit de la biographie d'un saint musulman du sud marocain surnommé Abū Yahyā asSā'ih (le « voyageur »).

C'est uniquement au début du XIV siècle que l'on assiste à un renouveau des sources arabes concernant les Canaries. Le cosmographe Šams ad-dīn ad-Dimasqi fait état d'une nouvelle expédition dans l'archipel, tandis qu'Ibn Khaldoun, dans ses Prolégomènes, 
décrit les mœurs de quelques indigènes canariens vendus comme esclaves sur les côtes marocaines. Il n'est pas étonnant de constater que ces serviteurs du sultan du Maroc aient été capturés, selon Ibn Khaldoun, par des chrétiens - des « francs » dans le texte -, les premières incursions européennes dans l'Archipel canarien étant contemporaines des faits rapportés par ce grand écrivain. Curieusement Ibn Khaldoun, généalogiste encyclopédique des Berbères et précurseur incontestable de la recherche historique moderne, passe sous silence l'ascendance berbère de ces esclaves canariens.

Les preuves archéologiques de la fréquentation du littoral canarien dont témoignent les sources classiques et arabes sont fragmentaires et difficiles à interpréter. L'origine romaine, longtemps acceptée sans réserve, de plusieurs amphores découvertes le long des côtes de Lanzarote et de l'îlot de La Graciosa est aujourd'hui contestée avec fermeté. Il s'agirait plutôt de poteries médiévales, voire d'époque moderne, dont l'origine est assurément ibérique. Mais l'existence de céramiques tournées associées à des contextes préhispaniques bien datés de Lanzarote et de la Grande Canarie et la découverte à Ténérife de vases indigènes imitant des prototypes antiques, constituent des arguments supplémentaires dignes d'être retenus dans le catalogue des données matérielles probables de ces navigations pré-européennes. Une dernière preuve significative dans ce domaine est fournie par la publication récente d'un ensemble d'inscriptions latines attestées à Fuerteventura et à Lanzarote. Ces textes épigraphiques proches de l'alphabet cursif pompéien, seraient à dater, d'après leurs inventeurs, entre le $\mathrm{I}^{\mathrm{er}}$ siècle av. J.-C. et le II siècle de l'ère chrétienne.

\section{Les Canaries dans l'orbite européenne : « redécouverte » et ethnohistoire}

$31 \mathrm{Au}$ cours $\mathrm{du} \mathrm{XVI} \mathrm{I}^{\mathrm{e}}$ siècle, les navigations des Vivaldi, l'arrivée aux Canaries du génois Lanceloto Malocello dont la carte nautico-géographique d'A. Dulcert (1339) fait état, l'expédition de la flotte portugaise commandée par le florentin Nicolosso Da Recco et, enfin, les voyages le long des côtes canario-sahariennes des majorquins, catalans et castillans entraînent la «redécouverte» de l'Archipel canarien par le monde occidental. Ces activités nautiques, qui ouvrent définitivement les routes atlantiques aux marines européennes, doivent être interprétées dans le cadre de l'effort croissant des puissances méditerranéennes pour court-circuiter le contrôle des musulmans sur les pistes caravanières du commerce de l'or.

32 L'ambitieux débarquement sur Lanzarote de Jean de Béthencourt en 1402 et les razzias continues des seigneurs des Canaries, héritiers des droits féodaux du gentilhomme normand, sont à l'origine de la conquête de plusieurs îles et de l'anéantissement d'une partie de leurs habitants, déjà décimés par les marchands d'esclaves européens. Mais ce n'est qu'en 1478 que les Rois Catholiques décident de prendre en charge la conclusion des opérations militaires en s'accordant avec des capitaines et des ecclésiastiques pour obtenir la soumission des territoires jusqu'alors non occupés : la Grande Canarie (1483), La Palma (1493) et Ténérife (1496). Dans ces îles les populations indigènes opposèrent la résistance la plus farouche à la pénétration castillane. Manifestement cette " politique africaine » des monarques espagnols, reléguée au second plan à cause de la guerre de succession et de la prise de Grenade, trouve son contexte précis au sein de la rivalité castillano-portugaise pour la domination de l'Atlantique. La conquête définitive des Canaries vise ainsi, dans l'esprit des Rois Catholiques, à l'obstruction des projets 
expansionnistes portugais, en leur bouchant une éventuelle escale africaine, et à l'établissement d'une tête de pont pour l'appropriation du Sahara atlantique et le soutien des expéditions castillanes vers la Guinée. Il semble donc évident que l'annexion de l'Archipel canarien, et par conséquent, la décomposition et assimilation du monde indigène, et les activités sur le littoral africain avoisinant, la « Mar Pequeña » de Berbérie des auteurs espagnols, sont deux phénomènes parallèles sinon complémentaires. Au Sahara, comme aux Canaries, les initiatives militaires et économiques privées sont supplantées par les entreprises officielles. En même temps on ne peut pas attribuer au hasard le fait que chacun des deux cycles de la conquête de l'archipel, seigneurial et royal, soit sanctionné par la construction d'une forteressefactorerie, telle que la « tour » de Santa Cruz de la Mar Pequena, sur la côte saharienne. Par ailleurs, le processus d'acculturation et assimilation des populations indigènes canariennes est parfaitement attesté dans leur participation aux incursions et entreprises continentales des colons hispano-canariens.

Jean de Bethencourt

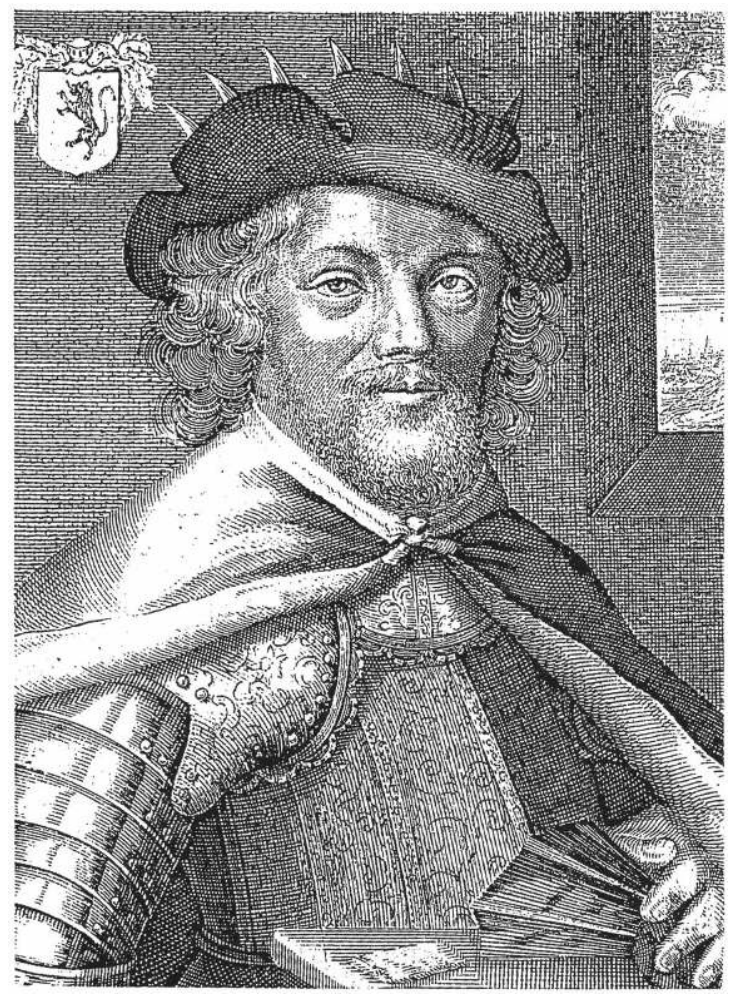

\section{Les sources ethnohistoriques}

Le contact puis l'affrontement des voyageurs et colons européens avec les indigènes canariens provoquèrent l'irruption de ces populations "exotiques" dans une littérature historique occidentale, dotée d'une curiosité ethnographique érudite tantôt naïve tantôt utilitaire, qui s'appliqua à dépeindre leurs mœurs parfois avec minutie. Mais la valeur des sources ethnohistoriques canariennes ne doit pas être néanmoins exagérée. Les renseignements tirés de ces répertoires, constitués pour l'essentiel de récits de voyages, chroniques de conquête, narrations savantes, rapports didactiques et documents administratifs postérieurs à la conclusion des opérations militaires, portent 
exclusivement sur la phase finale du peuplement préhispanique, bien que certaines des données que l'on y trouve plongent leurs racines dans des traditions assurément plus anciennes. De plus l'ethnocentrisme des auteurs, les problèmes d'identification et d'authentification des sources, la multiplicité des versions et, enfin, les avatars de la conservation de manuscrits, souvent connus par des copies tardives, réduisent la fiabilité de ces textes. Or, si le concours de l'Ethnohistoire s'avère fondamental afin de compléter de façon efficace la reconstitution des scénarios historiques des sociétés canariennes immédiatement préhispaniques, il n'est pourtant pas en mesure de supplanter, comme on l'a fait trop souvent, les données archéologiques et anthropologiques.

Les données fournies par l'ensemble des sources ethnohistoriques, relativement nombreuses et précises en ce qui concerne les îles de la Grande Canarie et de Ténérife, font référence aussi bien au milieu naturel qu'à l'aspect physique ou la démographie des populations préhispaniques. Elles apportent aussi des aperçus, d'intérêt inégal certes, d'ordre socio-économique où nombre d'auteurs puisent les termes insulaires d'un comparatisme linéaire, presque maladif, avec les berbérophones continentaux. D'après ces renseignements, il semblerait que le tissu social, la structure institutionnelle et l'organisation politique des diverses îles étaient singulièrement différents, atteignant leur degré de complexité le plus manifeste en Grande Canarie. Dans cette île, la concentration croissante du pouvoir politique et religieux en un seul lignage, au détriment des institutions oligarchiques tribales, témoigne d'une appropriation des moyens de production et, de ce fait, de la décomposition des groupes segmentaires traditionnels. L'émergence des chefferies canariennes telles que les cantons de Telde et Agaldar, dont l'origine et l'évolution sont difficiles à suivre en dehors des généalogies fantaisistes rapportées par les chroniqueurs, va sans doute de pair avec une modification de la territorialité politique insulaire et, par conséquent, des anciens systèmes d'alliances inter-tribales et des rapports sociaux de production. Quant à l'économie, les textes esquissent un tableau où le mode de production domestique et l'autarcie clanique tiennent une large place. L'élevage (mouton, chèvre, cochon, présence du chien) et parfois l'agriculture (blé, orge), qui attendent dans de nombreux cas la confirmation archéologique, coexistent avec la pêche et la cueillette (mollusques marins, baies, végétaux). D'autre part, les textes ethnohistoriques sont riches en précisions concernant la production artisanale, l'univers symbolique, la vie religieuse et les rites funéraires, les procédés de momification étant méticuleusement décrits dans plusieurs passages. La question toujours discutée de la technologie nautique des populations indigènes canariennes, et partant de l'existence de relations interinsulaires préhispaniques, peut être partiellement éclairée à l'aide d'un récit tardif dont la vraisemblance demeure confuse. Il s'agit du rapport adressé à la cour espagnole par L. Torriani, ingénieur de fortifications italien au service de Philippe II. Selon cet érudit les anciens habitants de la Grande Canarie possédaient les embarcations, creusées dans des troncs de dragonniers et gréées d'une voile de palme, qui leur permettaient de fréquenter les côtes de Ténérife et de Fuerteventura.

\section{Les origines légendaires et les berbères continentaux}

La recherche de la genèse, légendaire ou réelle, des indigènes des Canaries tient une place privilégiée au sein de la littérature ethnohistorique. Depuis le début de l'historiographie canarienne, aussi bien chroniqueurs et historiens que compilateurs 
s'appliquent à la détermination de la provenance des populations préhispaniques. Tour à tour, personnages bibliques, cananéens, perses, phéniciens ou romains ont été placés à l'origine du peuplement de l'archipel, tout en perpétuant une tradition solidement établie chez les généalogistes chrétiens, musulmans ou juifs de l'Afrique et de l'Europe médiévales. Mais les allusions textuelles à l'ascendance africaine, voire franchement berbère (termes alors sommairement équivalents dans l'imaginaire de l'Europe méditerranéenne), des anciens habitants des îles ne sont pas rares.

Parmi les récits contemporains de la conquête, ou immédiatement postérieurs à la conclusion des opérations militaires, seule la chronique dite d'Argüello, aujourd'hui disparue, fait référence à l'origine africaine probable des indigènes canariens. Argüello attribue le peuplement de l'ensemble des îles à des maurétaniens dont la révolte contre l'autorité romaine aurait entraîné la déportation aux Canaries ainsi que l'amputation de la langue. Comme J. Alvarez-Delgado l'a montré à juste titre, cette légende, connue sous plusieurs versions ultérieures, est de toute évidence une invention érudite hispano-canarienne qui tente d'expliquer les différences entre les diverses "langues » insulaires et leurs coïncidences lexicales avec les parlers berbères de la côte voisine. Il est très révélateur de signaler qu'aucune des versions de "Le Canarien", relatant les exploits des conquérants normands, ni les chroniques de Sedeño et Bernáldez, peutêtre écrites peu de temps après a soumission définitive des îles, ne mentionnent de façon explicite la filiation africaine des groupes préhispaniques canariens.

En revanche, les ouvrages des écrivains de la fin du Xvi et du XvII ${ }^{e}$ siècle attestent une multitude de références, plus ou moins artificielles, à l'arrivée aux Canaries d'effectifs berbères provenant du proche continent. Tel est le cas des Azanegh, la tribu placée par Torriani à l'origine du peuplement de Ténérife, dont le patronyme évoque celui des berbères Zenaga aujourd'hui cantonnés dans la partie occidentale du Trarza mauritanien. Les arguments fournis par ces auteurs sont tirés notamment de la proximité géographique et de certaines coïncidences d'ordre linguistique et culturel entre les habitants de l'aire canario-saharienne. Dans cet ensemble de textes ethnohistoriques, il est indubitable que les priorités chronologiques et l'enchaînement des « sources d'inspiration » de ces récits expliquent les similitudes et identités que l'on peut constater quant à l'apparentement «africain» des autochtones canariens. Mais ces «thèses africanistes" témoignent d'un choix idéologique délibéré à vocation essentiellement géopolitique. Il est vrai que les preuves géographiques et ethnographiques liées à l'expansion canario-andalouse sur le littoral saharien, dressaient $\mathrm{au} \mathrm{XVI} \mathrm{I}^{\mathrm{e}}$ siècle un canevas empirique où pouvait s'insérer des préoccupations «scientifiques " chères à une tradition humaniste qui recherchait les "origines » en ressuscitant les auteurs gréco-latins. Mais il n'en est pas moins certain que ces arguments servent d'emblée à légitimer un statu quo diplomatique. En effet, la filiation nord-africaine réelle ou présumée des anciens canariens, qui coexiste paradoxalement avec le mythe des indigènes grands, blonds et aux yeux clairs, vise à fonder les droits juridiques de la Couronne de Castille sur l'Archipel canarien et le littoral avoisinant. De façon éloquente, la contiguïté géographique et l'existence de liens historiques diffus entre la Péninsule ibérique, la Berbérie et les Iles Canaries furent mises en avant déjà au début $\mathrm{du} \mathrm{XIV}^{\mathrm{e}}$ siècle par le roi Alphonse XI dans le cadre de ses revendications territoriales africaines aussi précoces que démesurées. 


\section{La « langue » canarienne et la linguistique berbère}

parlers insulaires, la majorité des documents insistent sur la diversité linguistique et les problèmes d'inter-compréhension des populations des différentes îles. Même à l'intérieur des îles les plus étendues cette disparité linguistique a été aussi notée. Mais les renseignements textuels dont on dispose sont assez restreints et sujets à discussion. Sur le plan méthodologique la question la plus pertinente, assurément commune à toutes les approches diachroniques et glosso-chronologiques, est celle de la fiabilité des corpus lexicographiques. Peut-être n'est-il pas inutile de rappeler que les chroniqueurs et leurs copistes ultérieurs ont transcrit des mots et des phonèmes qui n'existaient pas dans une langue romane (italien, français et surtout castillan) elle-même différente de sa forme actuelle aussi bien du point de vue phonétique qu'orthographique. La multiplicité des notations des mots considérés équivalents, dont l'assimilation des «variantes» est parfois difficilement admissible, témoigne de façon expressive de la portée réelle de ces difficultés méthodologiques.

Indigènes des Canaries d'après L. Torriani (1592).

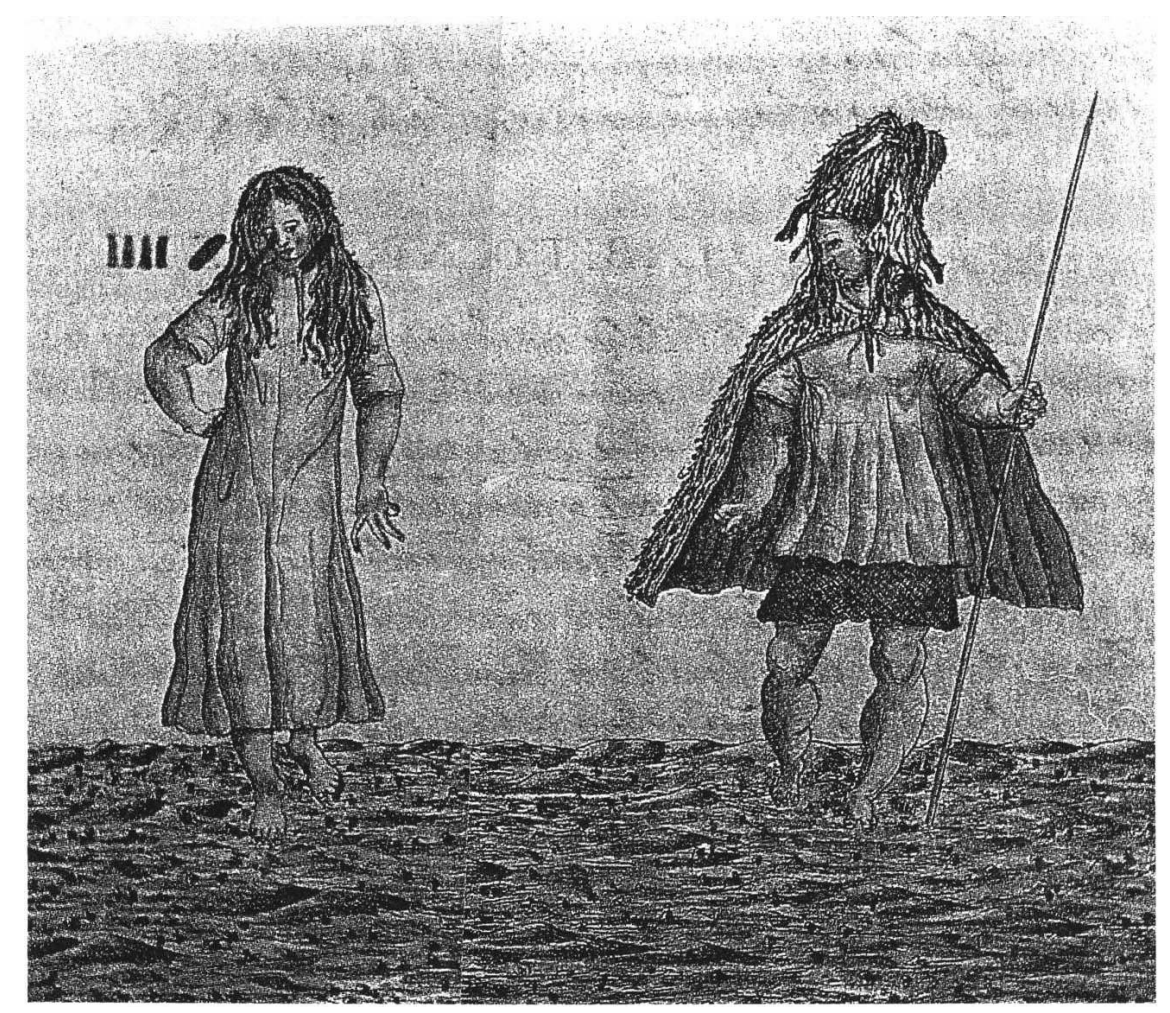

Les dialectes ou les langues parlés par les populations canariennes à l'arrivée des conquérants et colons européens, dont la connaissance est largement tributaire des travaux de G. Marcy, D.-J. Wölfel, J. Alvarez-Delgado et L. Galand, ont été généralement 
considérés comme un rameau du berbère. Mais, quoi qu'il en soit, il ne semble pas que le comparatisme réductionniste et souvent insensé, qui a fait fortune dans nombre d'études, puisse donner une réponse structurale satisfaisante à la totalité des problèmes linguistiques posés par l'étude des groupes préhispaniques canariens. C'est pour cela que des berbérisants réputés tels que L. Galand soutiennent, tout en reconnaissant l'existence d'affinités formelles, des avis plutôt critiques et sceptiques quant à l'apparentement berbère de l'ancienne «langue» canarienne. De toute évidence, l'importance relative des emprunts arabes relevés dans les répertoires lexicaux préhispaniques ne fait que confirmer, en dehors des hypothèses intuitives visant à identifier un substrat linguistique pré-berbère, la nécessité de faire appel à des modèles complexes et d'éviter l'établissement de parallelismes univoques.

Canariens se lançant un défi avant un duel, d'après L. Torriani (1592).

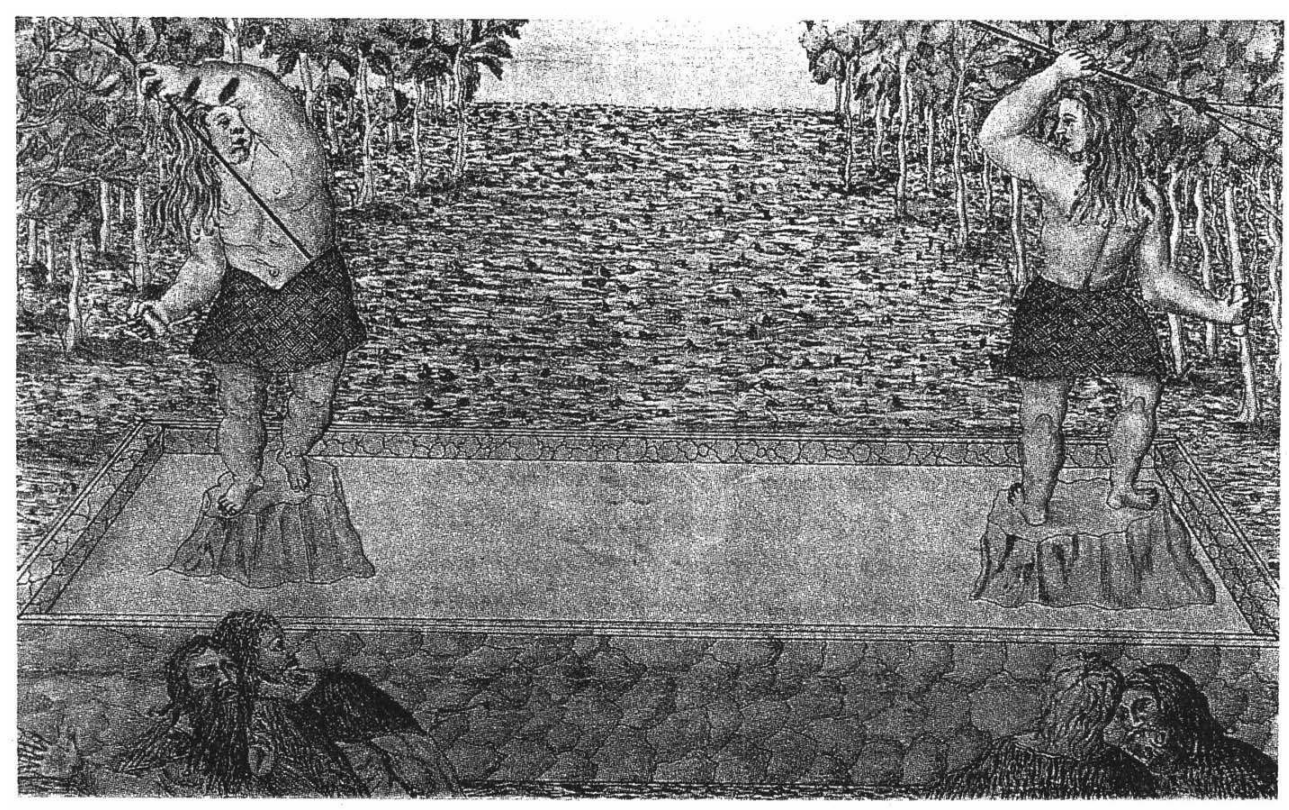

\section{BIBLIOGRAPHIE}

- Ouvrages généraux

BERTHELOT S. et BARKER-WEBB P., Histoire Naturelle des Iles Canaries, Plon-Béthume, Paris, 1839-1842.

BORY DE SAINT VINCENT J.B.G.M., Essais sur les îles Fortunées et l'antique Atlantide, ou précis de l'histoire générale de l'archipel des Canaries, Baudouin, Paris, 1803.

CASTRO ALFIN D., Historia de las Islas Canarias, De la prehistoria al descubrimiento, Editora Nacional, Madrid, 1983.

CHIL Y NARANJO G., Estudios históricos, climatológicos y patolôgicos de las Islas Canarias, D. Isidro

Miranda, Las Palmas, 1876.

Encyclopédie berbère, 11 | 1992 
GLASS G., The History of the Discovery and Conquest of the Canary Islands, R. \& J. Dodsley-T. Durham, London, 1764.

MARCY G, Une province lointaine du monde berbère, Les Iles Canaries, L'Archipel canarien et son histoire, Bull, de l'Enseignement public du Maroc (Rabat), 127 (1933), p. 170-191.

MERCER J., The Canary Islanders : their prehistory, conquest and survival, Rex Collings, London, 1980. MILLARES TORRES A., Historia General de las Islas Canarias, Edirca, Las Palmas, 1977.

VERNEAU R., Cinq années de séjour aux Iles Canaries, Bibliothèque de l'explorateur, A. Hennuyer, Paris, 1891.

VIERA Y CLAVIJO J., Noticias de la Historia General de las Islas Canarias, Goya ed., Sta. Cruz de Tenerife, 1982.

- Milieu physique et paléoenvironnement

AFONSO L. et al. (éds.), Geografia de Canarias, Ed. Interinsular Canaria, Sta. Cruz de Tenerife, 1988.

ARAÑA V. et CARRACEDO J.-C, Los volcanes de Canarias, I. Tenerife, II. Lanzarote y Fuerteventura, III. Gran Canaria, Ed. Rueda, Madrid, 1978-1979.

HANSEN MACHIN A., Los volcanes recientes de Gran Canaria, Excmo. Cabildo Insular de Gran Canaria-Ed. Rueda, Las Palmas-Madrid, 1987.

HUETZ DE LEMPS A., Le climat des Canaries, Publ. de la Fac. des Lettres et des Se. Humaines de ParisSorbonne, 54, Société d'Édition d'Enseignement Supérieur, Paris, 1969.

KUNKEL G. (éd.), Biogeography and Ecology in the Canary Islands, W. Junk, The Hague, 1976.

MECo J. et PETIT-MAIRE N. (éds.), El Cuatemario reciente de Canarias : Paleoclimatologia, Universidad de La Laguna-C.N.R.S., Las Palmas-Marseille, 1986.

PETIT-MAIRE N. et al., Paléoclimatologie des Canaries orientales (Fuerteventura), C.R. de l'Académie des Sciences de Paris (Paris), 303, s. II, 13, p. 1 241-1 246.

POMEL R.-S., Morphologie volcanique et paléoclimatologie des Iles Canaries, Comparaison avec d'autres milieux volcaniques insulaires (îles de la Mer Tyrrhénienne et de la Mer Egée, Ile de la Réunion), Thèse de Doctorat d'État de Géographie, Institut de Géographie, Université d'Aix-en-Provence, 1986.

ROGNON P. et COUDE-GAUSSEN G., Reconstitution paléoclimatique à partir des sédiments du Pléistocène supérieur et de l'Holocène du nord de Fuerteventura (Canaries), Zeitschrift fur Géomorphologie, n.f., 31, 1, p. 1-19.

- Préhistoire

ARCO AGUILAR M.C. del et Navarro Mederos J.-F., Los aborigènes, Historia popular de Canarias, I, Centro de la Cultura Popular Canaria, Sta. Cruz de Tenerife, 1987.

ATOCHE PEÑA P. et al., Elyacimiento arqueológico de «El Bebedero » (Teguise, Lanzarote), Resultados de la primera campana de excavaciones, Universidad de La Laguna-Ayuntamiento de Teguise, La LagunaTeguise, 1989.

BALBIN BEHRMANN R. de et al., Lanzarote prehispánico, Notas para su estudio, Actas del XVIII Congreso Nacional de Arqueologia (Canarias, 1985), Zaragoza, 1987, p. 19-49.

BALOUT L, Réflexions sur le problème du peuplement préhistorique de l'Archipel canarien, Anuario de Estudios Atlânticos (Madrid-Las Palmas), 15 (1969), p. 133-145. 
BELTRAN MARTINEZ A., Los grabados del Barranco de Balos (Gran Canaria), El Museo Canario-Patronato J.M. Quadrado, Las Palmas, 1971.

Beltran A. et Alzola J.-M., La Cueva Pintada de Gaïdar, Monografias Arqueológicas, 17, Zaragoza, 1974.

BERTHELOT S., Antiquités canariennes ou annotations sur l'origine des peuples qui occupèrent les îles Fortunées, depuis les premiers temps jusqu'à l'époque de leur conquête, Pion, Paris, 1879.

CABRERA PEREZ J.-C, Los majos, Poblacion prehistóriea de Lanzarote, Col. Rubicón, Excmo. Cabildo Insular ed lanzarote, Arrecife, 1989.

CAMPS G., L'Homme de Mechta El-Arbi et sa civilisation, Contribution à l'étude des origines guanches, Anuario de Estudios Atlánticos (Madrid-Las Palmas), 15 (1969), p. 257-262.

DIEGo cuscoY L, Los Guanches, Viday cultura delprimitivo habitante de Tenerife, Publ. del Museo

Arqueológico, 7, Sta. Cruz de Tenerife, 1968.

DIEGo CUSCOY L., Gánigo, Estudio de la cerámica de Tenerife, Publ. del Museo Arqueológico, 8, Sta. Cruz de Tenerife, 1971.

GARCIA Y BELLIDO A., Sobre las ánforas antiguas de Canarias, Homenaje a Elias Serra Ráfols, La Laguna, 1970, t. II, p. 193-199.

GONZALEZ ANTON R., Las cerámicas aborigènes canarias, Col. Guagua, Mancomunidad de Cabildos, Plan Cultural y Museo Canario, Las Palmas, 1980.

GONZALEZ ANTON R. et TEJERA GASPAR A., Los aborigènes canarios, Gran Canaria y Tenerife, Col. Minor, 1, Universidad de La Laguna, La Laguna, 1981.

HERNANDEZ PEREZ M.-S., La Palma prehispánica, El Museo Canario, Las Palmas, 1977.

ноотоN E.-A., The ancient inhabitants of the Canary Islands, Harvard African Studies, VII, Cambridge, Mass., 1925.

Investigaciones Arqueológicas en Canarias (Las Palmas), I (1988), II (1990).

JIMENEZ GOMEZ M.-C, Aproximación a la Prehistoria de El Hierro, Fundación J. March, Série

Universitaria, 177, Madrid, 1982.

JIMENEZ GONZALEZ J.-J., Los Canarios, Etnohistoria y Arqueologia, ACT-Museo Arqueológico, 14, Sta. Cruz de Tenerife, 1990.

MARTIN DE GUZMAN C, Las culturas prehistôricas de Gran Canaria, Excmo. Cabildo Insular de Gran Canaria, Madrid-Las Palmas, 1984.

MARTIN DE GUZMAN C, Los problemas de la navegaciôn pre y protohistôrica en el Mar de Canarias y la fachada atlántico-sahariana, Actas del V Coloquio de Historia Canario-Americana (Las Palmas, 1982), Excma. Mancomunidad Provincial Interinsular de Cabildos de Las Palmas-Excmo. Cabildo Insular de Gran Canaria, Las Palmas, 1986, t. IV, p. 27-144.

NAVARRo MEDEROS J.-F., Prehistoria de la isla de La Gomera, Col. Guagua, Mancomunidad de Cabildos, Plan Cultural y Museo Canario, Las Palmas, 1981.

ONRUBIA-PINTADO J., Les relations entre les Iles Canaries et l'Afrique du Nord pendant les temps préhispaniques, Archéologie et Ethnohistoire d'une aire marginale, Travaux du L.A.P.M.O. (Aix-enProvence), 1985, ét. 11.

ONRUBIA-PINTADO J., Les cultures préhistoriques des Iles Canaries, État de la question,

L'Anthropologie (Paris), 91 (1987), p. 653-678. 
PELLICER M., Elementos culturales de la prehistoria canaria (Ensayo sobre origenes y cronología de las culturas), Miscelánea Arqueológica, Barcelona, 1974, II, p. 145-161.

PERICOT L., Algunos nuevos aspectos de los problemas de la Prehistoria canaria, Anuario de Estudios Atlánticos (Madrid-Las Palmas), 1 (1955), p. 579-619.

SERRA RAFOLS E., Les relations possibles des cultures canariennes avec celles de l'ouest Africain, Actas del V Congr. Panafr. de Prehistoria y de estudio del Cuatemario, Publ. del Museo Arqueológico, 6, Sta. Cruz de Tenerife, 1966, p. 245-247.

SOUVILLE G., Remarques sur le problème des relations entre l'Afrique du Nord et les Canaries au Néolithique, Anuario de Estudios Atlánticos (Madrid-Las Palmas), 15 (1969), p. 367-383.

TARRADELl M., Los diversos horizontes de la Prehistoria canaria, Anuario de Estudios Atlánticos (Madrid-Las Palmas), 15 (1969), p. 385-391.

TEJERA GASPAR A. et GONZALEZ ANTON R., Las culturas aborigenes canarias, Interinsular-Ed. Canarias, Sta. Cruz de Tenerife, 1987.

ZEUNER F.-E., Summary ofthe cultural problems of the Canary Islands, Actas del V Congr. Panafr. de Prehistoria y de estudio del Cuatemario, Publ. del Museo Arqueológico, 6, Sta. Cruz de Tenerife, 1966, p. 277-288.

- Paléoanthropologie

BERMUDEZ DE CASTRO J.-M., La dentición de los pobladores prehistóricos de las Islas Canarias, Estudio anthropológico, Thèse de Doctorat, Universidad Complutense, Madrid, 1985.

BILLY G., Le peuplement préhistorique de l'Archipel canarien, El Museo Canario (Las Palmas), XLI (1980-1981), p. 59-74.

FUSTE M., Contribution à l'Anthropologie de la Grande Canarie, L'Anthropologie (Paris), 63 (1959), p. 295-318.

FUSTE M., Aperçu sur l'Anthropologie des populations préhistoriques des Iles Canaries, Actas del V Congr. Panafir. de Prehistoria y de estudio del Cuaternario, Publ. del Museo Arqueológico, 6, Sta. Cruz de Tenerife, 1966, p. 69-79.

onRUBiA-PINTADo J. et DUtOUR O., Les Cro-Magnons aux Iles Canaries, Les Dossiers d'Archéologie (Dijon), 161 (1991), p. 74-81.

SCHWIDETZKY I., La población prehispánica de las Islas Canarias, Investigaciones antropológicas, Publ. del Museo Arquelógico, 4, Sta. Cruz de Tenerife, 1963.

SCHWIDETZKY I., Population biology of the Canary Islands, El Museo Canario (Las Palmas), XLI

(1980-1981), p. 47-56.

VALLOIS H.-V., Les hommes de Cro-Magnon et les guanches : les faits acquis et les hypothèses, Anuario de Estudios Atlánticos (Madrid-Las Palmas), 15 (1969), p. 97-119.

VERNEAU P., Rapport d'une mission scientifique dans l'Archipel Canarien, Archives des Missions Se. et Littéraires (Paris), $3^{\mathrm{e}}$ sér., XVIII (1887), p. 567-817.

- Sources classiques et arabes

BLAZQUEZ J.-M., Las Islas Canarias en la Antiguedad, Anuario de Estudios Atlánticos (Madrid-Las Palmas), 23 (1977), p. 35-50.

CABRERA PERERA A., Las Islas Canarias en el Mundo Clásico, Viceconsejeria de Cultura y Déportes, Gobierno de Canarias, Las Palmas, 1988. 
CORNEVIN R., Voyages musulmans aux Canaries du XIII ${ }^{\mathrm{e}}$ siècle, Notes Africaines (Dakar), 96 (1962), p. 128.

DESANGES J., Recherches sur l'activité des méditerranéens aux confins de l'Afrique (VIe siècle av. J.-C. - IV siècle ap. J.-C), Coll. de l'École Française de Rome, 38, EFR, Rome, 1978.

LEWICKI T., Encore sur les voyages arabes aux Canaries au Moyen-Age, Études maghrébines et soudanaises, II, Éd. Scientifiques de Pologne, Varsovie, 1983, p. 9-31.

MAUNY R., Les navigations médiévales sur les côtes sahariennes antérieures à la découverte portugaise, Centro de Estudios Histôricos Ultramarinos, Lisboa, 1960.

MAUNY R., Navigations arabes anonymes aux Canaries du XII ${ }^{\mathrm{e}}$ siècle, Notes Africaines (Dakar), 106 (1965), p. 61.

REBUFFAT R, Arva beata petamus arva divites et insulas, Mélanges offerts à J. Heurgon, L'Italie préromaine et la Rome républicaine, Coll. de l'École Française de Rome, 27, Rome, 1976, p. 877-902. schmiTT P., Connaissance des îles Canaries dans l'Antiquité, Latomus (Bruxelles), XXVII (1968), p. 362-391.

- Redécouverte européenne et textes ethnohistoriques ABREU GAUNDO Fr. J. de, Historia de la conquista de las siete islas de Canaria, Goya éd., Sta. Cruz de Tenerife, 1977.

ALVAREZ DELGADO J., Leyenda erudita sobre la población de Canarias con africanos de lenguas cordadas, Anuario de Estudios Atlánticos (Madrid-Las Palmas), 23 (1977), p. 51-81.

AZNAR VALLEJO E., La integración de las Islas Canarias en la Corona de Castilla (1478-1526), Col. Viera y Clavijo, VI, Universidad de Sevilla-Universidad de La Laguna, Sevilla-La Laguna, 1983.

BETHENCOURT J. de, Le Canarien, Livre de la conquête et conversion des Canaries (1402-1422), Publ. Gabriel Gravier, Rouen, 1874.

CA'DA Mosto A. da, Relation des voyages à la côte occidentale d'Afrique (1455-1457), E. Leroux, Paris, 1895. CENIVAL P. de et MONOD Th., Description de la côte d'Afrique de Ceuta au Sénégal par Valentin Femandes (1506-1507), Publ. du Comité d'Et. Hist. et Sc. de l'A.O.F., sér. A, VI, Paris, 1938.

DUPUIS J., Les Iles Canaries avant la conquête espagnole (d'après le Manuscrit de Torriani), Cah. du Monde Hispanique et Luso-Brésilien (Toulouse), 33 (1979), p. 91-102.

EANNES DE ZURARA G, Chronique de Guinée, I.F.AN., Dakar, 1960.

ESPINOSA Fr. A. de, Historia de Nuestra Senora de Candelaria, Goya Ed., 1967.

FALL Y.-K., L'Afrique à la naissance de la cartographie moderne, Les cartes majorquines : XIVe-XVe siècles, Karthala-C.RA., Paris, 1982.

Le Canarien, Cronicas francesas de la conquista de Canarias, ACT-Excmo. Cabildo Insular de Tenerife, Sta. Cruz de Tenerife, 1986.

MAUNY R., Les navigations médiévales sur les côtes sahariennes antérieures à la découverte portugaise (1434), Centro de Estudios Históricos Ultramarinos, Lisboa, 1960.

MORALES PADRON F., Canarias : Crónicas de su conquista, Excmo. Ayuntamiento de Las Palmas-El Museo Canario, Las Palmas, 1978.

RUMEU DE ARMAS A., Espana en el Africa Atlántica, I.D.E.A., C.S.I.C., Madrid, 1956. 
RUMEU DE ARMAS A., El obispado de Telde, Misioneros mallorquines y catalanes en el Atlántico, Ayuntamiento de Telde, Madrid-Telde, 1986.

TORRIANI L., Descripción e Historia del reino de las Islas Canarias antes Afortunadas con el parecer de sus fortificaciones, Goya éd., Sta. Cruz de Tenerife, 1978.

- Paléo-linguistique et inscriptions libyques

ALVAREZ DELGADO J., Inscripciones libicas de Canarias, Ensayo de interpetación libica, Universidad de La Laguna, Tenerife, 1964.

ALVAREZ Delgado J., Antropónimos Guanches, Col. Guagua, Mancomunidad de Cabildos, Plan Cultural y Museo Canario, Las Palmas, 1979.

CAMPS G., Recherches sur les plus anciennes inscriptions libyques de l'Afrique du Nord et du Sahara, Bull. Archéologique du C.T.H.S. (Paris), n.s. 10-11/1974-1975 (1978), p. 143-166.

CHAKER S., Textes en linguistique berbère (Introduction au domaine berbère), Éd. du C.N.R.S., Paris, 1984. DIEGO CUSCOY L. et GALAND L., Nouveaux documents des Iles Canaries, L'Anthropologie (Paris), 79 (1975), p. 5-37.

GALAND L., Die afirkanischen und kanarischen Inschriften des libyschberberischen typus, Almogaren (Hallein), IV (1973), p. 65-79.

GALAND L., Langue et littérature berbères, Vingt-cinq ans d'études, C.R.E.S.M., C.N.R.S., Paris, 1979.

GALAND L., El beréber una clave para el canario ?, Eres (Sta. Cruz de Tenerife) v.l, 1 (1990), p. 87-93. HERNANDEZ R. et SPRTNGER R., Hallazgo de nuevas inscripciones aborigènes en la Isla de El Hierro, Almogaren (Hallein), XI-XII (1980-1981), p. 15-25.

MARCY G., Notas sobre algunos topónimos y nombres antiguos de tribus bereberes en las Islas Canarias, Anuario de Estudios Atlânticos (Madrid-Las Palmas), 8 (1962), p. 239-289.

MILITARIEV A., Tamâhaq Tuaregs in the Canary Islands (Linguistic Evidence), Aula Orientalis, 6 (1988), p. 195-196.

NAVARRO ARTILES F., Teberite, Diccionario de la lengua aborigen canaria, Edirca, Las Palmas, 1981.

VYCICHL W., Les Berbères des Iles Canaries, Éléments historiques et linguistiques, Études et Documents Berbères (Paris), 2 (1987), p. 42-62.

WOLFEL D.-J., Le problème des rapports du guanche et du berbère, Hespéris (Paris), XL (1953), p. 523-527.

WOLFEL D.-J., Monumenta Linguae Canariae, Die Kanarischen Sprachdenkmäler, Akademische Druck. u. Verlagsanstalt, Graz, 1965.

\section{INDEX}

Mots-clés : Antiquité, Canaries (Îles), Géographie, Origines, Protohistoire, Tribus 